\title{
Inward-facing conformation of the zinc transporter YiiP revealed by cryoelectron microscopy
}

\author{
Nicolas Coudray ${ }^{a}$, Salvatore Valvo ${ }^{b}$, Minghui Hua , Ralph Lasalaa , Changki Kim ${ }^{a}$, Martin Vink ${ }^{a, c}$, Ming Zhou ${ }^{d}$, \\ Davide Provasic, Marta Filizolac, Juoehi Tao ${ }^{\mathrm{e}}$, Jia Fang ${ }^{\mathrm{e}}$, Pawel A. Penczek ${ }^{\mathrm{e}}$, Iban Ubarretxena-Belandiac, \\ and David L. Stokes ${ }^{\mathrm{a}, \mathrm{b}, 1}$
}

\begin{abstract}
aLaboratory of Cryo-Electron Microscopy, New York Structural Biology Center, New York, NY 10027; ' Skirball Institute, Department of Cell Biology, New York University School of Medicine, New York, NY 10016; 'Department of Structural and Chemical Biology, Icahn School of Medicine at Mt. Sinai, New York, NY

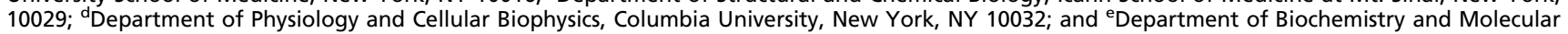
Biology, University of Texas-Houston Medical School, Houston, TX 77030

Edited by Wah Chiu, Baylor College of Medicine, Houston, TX, and approved December 26, 2012 (received for review September 5, 2012)

\begin{abstract}
YiiP is a dimeric $\mathrm{Zn}^{2+} / \mathrm{H}^{+}$antiporter from Escherichia coli belonging to the cation diffusion facilitator family. We used cryoelectron microscopy to determine a 13-Å resolution structure of a YiiP homolog from Shewanella oneidensis within a lipid bilayer in the absence of $\mathrm{Zn}^{2+}$. Starting from the X-ray structure in the presence of $\mathrm{Zn}^{2+}$, we used molecular dynamics flexible fitting to build a model consistent with our map. Comparison of the structures suggests a conformational change that involves pivoting of a transmembrane, fourhelix bundle (M1, M2, M4, and M5) relative to the M3-M6 helix pair. Although accessibility of transport sites in the X-ray model indicates that it represents an outward-facing state, our model is consistent with an inward-facing state, suggesting that the conformational change is relevant to the alternating access mechanism for transport. Molecular dynamics simulation of YiiP in a lipid environment was used to address the feasibility of this conformational change. Association of the C-terminal domains is the same in both states, and we speculate that this association is responsible for stabilizing the dimer that, in turn, may coordinate the rearrangement of the transmembrane helices.
\end{abstract}

membrane protein | secondary transporter | zinc antiporter | FieF

$\mathbf{T}^{\mathrm{s}}$ ransition metal ions play either structural or functional roles for approximately half of all cellular proteins (1). Specifically, transition metal ions, such as $\mathrm{Cu}^{+}, \mathrm{Zn}^{2+}, \mathrm{Fe}^{2+}$, and $\mathrm{Co}^{2+}$, serve as essential cofactors, and their relative concentrations and availability are carefully controlled to ensure that a given protein is loaded with the appropriate metal ion. Heavy metals, such as $\mathrm{Cd}^{2+}, \mathrm{Hg}^{2+}$, and $\mathrm{Pb}^{3+}$, often bind these proteins with high affinity and thus displace the physiologically relevant metal ion. To cope with the complexity of this situation, the cell uses an extensive array of pumps, transporters, and binding proteins, all of which have distinct functions in regulating the distribution of the various metal ions.

In the case of $\mathrm{Zn}^{2+}$, homeostasis relies on membrane transporters from a number of different protein families. Primary transport involves ATP-binding cassette transporters (e.g., ZnuB/C) and P-type pumps (e.g., ZntA, ZnuA), which directly couple ATP hydrolysis to the import and export of $\mathrm{Zn}^{2+}$, respectively (2). Secondary transporters come from the family of Zrt, Irt-like proteins (3) and the Nramp broad-spectrum cation transporters (2), both of which are implicated in $\mathrm{Zn}^{2+}$ import.

In the present study, we examined YiiP, which belongs to the cation diffusion facilitator (CDF) family and is responsible for the export of $\mathrm{Zn}^{2+}(4,5)$. The CDF family also includes the bacterial homologs $\mathrm{ZitB}$ and $\mathrm{CzrB}$, as well as eight human homologs designated $\mathrm{Znt1}-8$. Znt3 is associated with loading of $\mathrm{Zn}^{2+}$ into synaptic vesicles, and $\mathrm{Znt} 8$ mediates $\mathrm{Zn}^{2+}$ accumulation by insulin-containing secretory vesicles in pancreatic $\beta$ cells and thus is associated with type 2 diabetes (6). YiiP was first identified in Escherichia coli, and early studies indicated an association with $\mathrm{Fe}^{2+}$ transport; thus the name FieF (7). However, subsequent studies showed YiiP to be a very efficient transporter of $\mathrm{Zn}^{2+}$ and $\mathrm{Cd}^{2+}$, but not of $\mathrm{Fe}^{2+}, \mathrm{Co}^{2+}, \mathrm{Cu}^{2+}, \mathrm{Mn}^{2+}$, or $\mathrm{Ni}^{2+}(8$, 9). YiiP was demonstrated to be dimeric both in detergent solutions and in the membrane (10). X-ray crystallography revealed a V-shaped dimer with closely apposed C-terminal domains, but with membrane domains separated by $\sim 20 \AA$. The dimer is stabilized by four $\mathrm{Zn}^{2+}$ ions mediating a tight interface between cytoplasmic $\mathrm{C}$-terminal domains and a conserved salt bridge near the membrane surface $(11,12)$. The transmembrane domain consists of six helices grouped into a bundle of four (M1, M2, M4, and M5) and a bundle of two (M3 and M6). One $\mathrm{Zn}^{2+}$ ion is independently bound within the four-helix bundle of each monomer, tetrahedrally coordinated by three aspartates and one histidine. In the mammalian homologs, substitution of one of the aspartate residues with a second histidine eliminated their ability to transport $\mathrm{Cd}^{2+}(9)$, thus making them $\mathrm{Zn}^{2+}$-specific transporters.

In the conformation adopted by this X-ray structure, the transport sites were accessible from the periplasmic side of the membrane, consistent with an outward-facing state described by the alternating access mechanism of transport (13). FRET studies suggested a model for transport involving a scissoring of the dimer about the salt bridge, leading to $\mathrm{Zn}^{2+}$ release from sites both in the membrane and between the C-terminal domains (12).

We used electron crystallography as an alternative to X-ray crystallography for studying the structure of YiiP within a lipid bilayer (14). We found that the homolog from Shewanella oneidensis formed narrow, helical crystals, which were imaged by cryoelectron microscopy (cryo-EM) and used to determine the $3 \mathrm{D}$ structure. Based on transport assays and on the $\mathrm{Zn}^{2+}$ dependence of crystallization, we concluded that our structure represented the conformation with transport sites occupied by $\mathrm{H}^{+}$ instead of $\mathrm{Zn}^{2+}$. We used molecular dynamics (MD) to fit an atomic model to our electron crystallographic density map and to compare this model with the previous structure by X-ray crystallography. This comparison suggests that the EM structure corresponds to the inward-facing state and offers mechanistic insights into the structural basis for the alternating access model of transport.

Author contributions: M.Z., M.F., P.A.P., I.U.-B., and D.L.S. designed research; N.C., S.V., M.H R.L., C.K., M.V., D.P., J.T., J.F., and D.L.S. performed research; M.Z. contributed new reagents/ analytic tools; N.C., S.V., M.H., R.L., C.K., M.V., D.P., M.F., J.T., J.F., P.A.P., I.U.-B., and D.L.S analyzed data; and N.C., M.F., P.A.P., I.U.-B., and D.L.S. wrote the paper.

The authors declare no conflict of interest.

This article is a PNAS Direct Submission.

Data deposition: The atomic coordinates and structure factors have been deposited in the Protein Data Bank, www.pdb.org (PDB ID codes EMD-5450 and 3J1Z).

${ }^{1}$ To whom correspondence should be addressed. E-mail: stokes@nyu.edu.

This article contains supporting information online at www.pnas.org/lookup/suppl/doi:10. 1073/pnas.1215455110/-/DCSupplemental. 


\section{Results}

2D Crystallization. The homolog of YiiP from S. oneidensis was identified by the New York Consortium on Membrane Protein Structure as one of the Protein Structure Initiative targets to be highly expressed in E. coli and stable in detergent solution (15). After purification using conditions defined by the New York Consortium on Membrane Protein Structure, we employed a highthroughput approach to systematically test a wide range of parameters relevant to 2D crystallization (16), namely lipid species, lipid:protein ratio, $\mathrm{pH}$, and temperature. A 96-well dialysis block was used to remove detergent from each of the conditions (17), and the samples were negatively stained and screened robotically by EM (18). Narrow tubular crystals formed readily in dioleoylphosphatidyl glycerol (DOPG) after $\sim 5 \mathrm{~d}$ (Fig. S1) over a wide range of lipid:protein ratios ( $0.25-1.5$ by weight).

Although we screened a wide range of lipids and buffers, we were not able to obtain planar 2D crystals more suitable for analysis at high resolution. Such behavior is consistent with the idea that molecular interactions between YiiP molecules dictate the tubular morphology, and that the lipid molecules are relatively passive participants in the crystallization process. Indeed, tubular crystals were observed with other lipids used in our screen, although their abundance and order were lower than those obtained with DOPG.

$\mathrm{Zn}^{2+}$ Dependence of Crystallization and Transport. To characterize the transport function of the YiiP homolog from $S$. oneidensis and to assess its conformational state within the helical crystals, we measured $\mathrm{Zn}^{2+}$ and $\mathrm{Cd}^{2+}$ uptake into liposomes. Similar to previous studies on the E. coli homolog (8), we trapped a fluorescent indicator inside reconstituted liposomes and monitored the timedependent influx of both $\mathrm{Zn}^{2+}$ and $\mathrm{Cd}^{2+}$. Kinetic analysis of these data indicated $\mathrm{K}_{0.5}$ values of $380 \mu \mathrm{M}$ and $150 \mu \mathrm{M}$, respectively (Fig. S2). A distinct pH dependence of transport was evident, with maximal transport at $\mathrm{pH} 7.8$ and marked inhibition at higher $\mathrm{pH}$, consistent with the countertransport of $\mathrm{H}^{+}$previously demonstrated with ZitB (19).

We then studied the $\mathrm{Zn}^{2+}$ dependence of crystallization. Although $\mathrm{Zn}^{2+}$ was not added during the crystallization of YiiP, the existence of very-high-affinity sites $(20,21)$ raises the possibility that residual $\mathrm{Zn}^{2+}$ might remain bound to the protein. Thus, we used citrate and $N$-(2-acetamido)iminodiacetic acid to buffer the concentration of free $\mathrm{Zn}^{2+}$ over a wide range of concentrations $(10 \mathrm{nM}-1 \mathrm{mM})$ during crystallization trials. In addition, EDTA, which has a much higher affinity for $\mathrm{Zn}^{2+}$, was used to ensure the absence of free $\mathrm{Zn}^{2+}$ in the solutions.

These studies showed that YiiP produced long helical tubes when free $\mathrm{Zn}^{2+}$ concentrations were below $10 \mu \mathrm{M}$, but that no tubes grew at concentrations above $100 \mu \mathrm{M}$ (Fig. S3). Short, poorly ordered tubes were observed at intermediate concentrations. The addition of $\mathrm{Cd}^{2+}$ and $\mathrm{Fe}^{2+}$ had similar effects on crystallization. Tubes also grew in the presence of $0.5 \mathrm{mM}$ EDTA; however, similar to previous reports on the E. coli homolog (21), extended incubation in $5 \mathrm{mM}$ EDTA led to aggregation of the protein, suggesting that binding of $\mathrm{Zn}^{2+}$ to very-high-affinity sites may serve to stabilize the protein fold.

3D Structure of Yiip from Helical Crystals. We used two different approaches to calculate the 3D structure of YiiP from images of the helical crystals. Specifically, iterative helical real-space reconstruction (IHRSR) (22) and classical Fourier-Bessel (FB) (23) reconstruction methods were used to independently determine density maps. Comparison of the two structures provided a validation of structural features in the resulting maps and allowed us to assess the relative efficiency of the two approaches. Both methods rely on indexing the helical symmetry, which was done by comparing the positions of individual layer lines in Fourier space with the outer radius of the tube in real space $(136 \AA)$. Assignment of Bessel orders for individual layer lines (Fig. S1) and an approximate repeat distance along the helical axis of 3,320 $\AA$ allowed us to calculate a twist $(\Delta \varphi)$ of $56.4^{\circ}$ and a rise $(\Delta z)$ of $17.1 \AA$ for the fundamental helix, which is augmented with threefold rotational symmetry around the helical axis (C3 symmetry).

In addition, the phases along the layer lines provide evidence of a twofold axis lying normal to the helical axis and the resulting D3 symmetry was applied during the reconstruction. For both reconstructions, an envelope function or temperature factor correction was estimated by building an atomic model of the helical assembly based on the X-ray structure for YiiP and comparing its spherically averaged power spectrum with that derived from the experimental map. Both Fourier shell coefficients from IHRSR and twofold related phase residuals for FB reconstruction indicate a resolution of $\sim 13 \AA$ (Fig. 1). Features of the two maps were largely identical, although definition of protein density within the membrane were somewhat better for the IHRSR map (Fig. S4).

The resulting cylindrical structure (Fig. 1) shows discrete densities at an outer radius $(\sim 110 \AA)$, along with a relatively continuous cylinder of density between radii of $40 \AA$ and $80 \AA$. This continuous cylinder has somewhat higher density in the radially projected profile (Fig. $1 C$ ) and harbors distinct peaks at $43 \AA$ and $70 \AA$. This profile is characteristic of helical reconstructions, with peaks likely corresponding to the phosphate head groups at the boundaries of the lipid bilayer. Indeed, the spacing of these peaks is consistent with the $27 \AA$ thickness of the hydrocarbon core of 18-carbon, monounsaturated lipids (24), such as the DOPG used to produce the helical crystals.

Fitting X-Ray Structure to Map from Helical Crystals. Although our EM map was generally consistent with the positions of the transmembrane and $\mathrm{C}$-terminal domains in the X-ray structure,
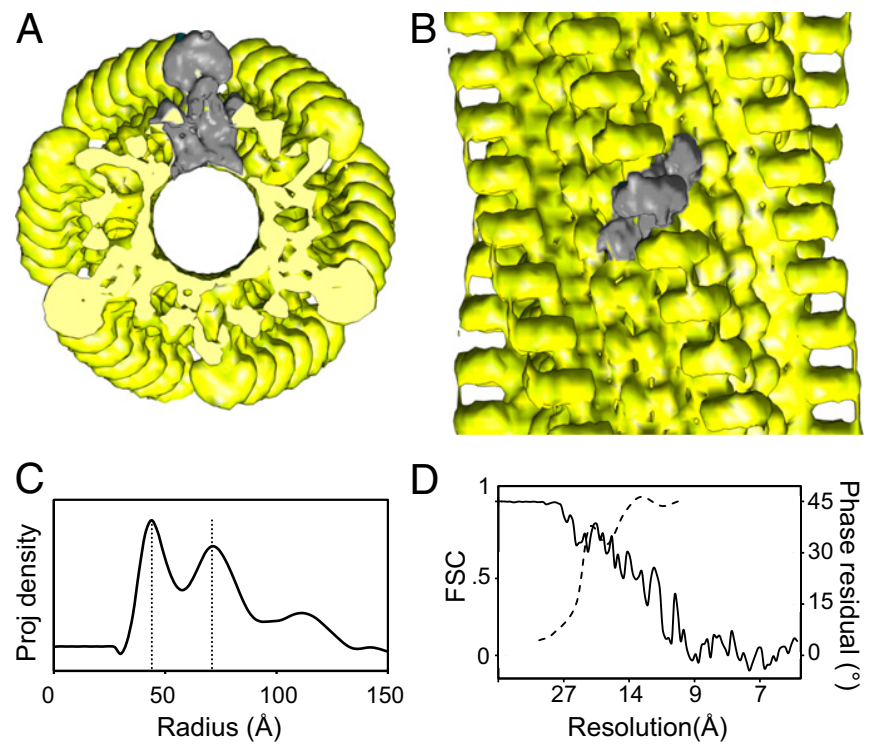

Fig. 1. Helical reconstruction of YiiP. $(A$ and $B)$ Views parallel and perpendicular to the helical axis showing helical packing of dimers. A single dimeric unit is shaded in gray. $(C)$ Projected radial density distribution for the reconstruction showing peaks at $43 \AA$ and $70 \AA$ (dotted lines), corresponding to the position of phosphate head groups of the lipid bilayer. Densities at higher radius $(\sim 110 \AA)$ are associated to the C-terminal domains that protrude from the cylindrical structure. $(D)$ The resolution of the reconstruction assessed by the Fourier shell coefficient for IHRSR tube (solid line) and twofold phase residuals for the FB reconstruction (dashed line). Both are consistent with a resolution of $\sim 13 \AA$ ( 0.5 criterion for the Fourier shell coefficient and $40^{\circ}$ for twofold residuals). 

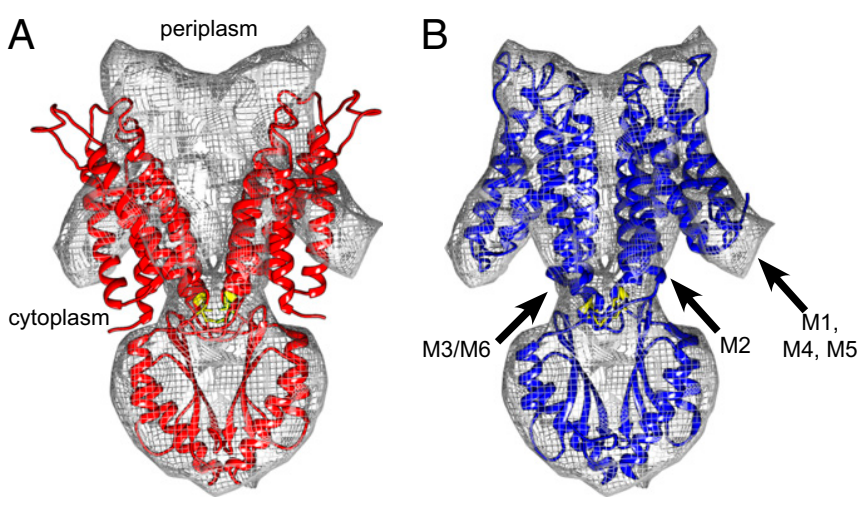

Fig. 2. Fitting an atomic model to the helical reconstruction. $(A)$ Rigid body docking of the X-ray structure for YiiP (PDB ID code 3H90) to the IHRSR map. The C-terminal domain fits snugly in density protruding from the helical tubes, but the $\mathrm{Y}$-shaped transmembrane domain must undergo major changes to match the map. ( $B$ ) Results of MDFF fitting of a homology model to the IHRSR map. The transmembrane regions of the monomers have moved closer together, and the cytoplasmic part of the M1-M2-M4-M5 bundle of transmembrane helices has moved away from the M3-M6 pair. The $\mathrm{N}$ terminus of YiiP in the X-ray structure is disordered, and the MDFF model includes the slightly longer $\mathrm{N}$ terminus from the $\mathrm{S}$. oneidensis homolog, which fits nicely into the corresponding density at the cytoplasmic surface of the membrane (under the arrowheads indicating the location of M1, M4, and M5). Residues composing the conserved salt bridge, termed the charge interlock, are shown in yellow near the cytoplasmic membrane surface.

juxtaposition of this structure with the map indicated that YiiP adopted a different conformation (Fig. $2 A$ ). Sequence comparisons between the $E$. coli protein used for the X-ray structure and the $S$. oneidensis protein used here (Fig. S5) indicate that they have a high degree of sequence similarity (46\% identity) with conservation of all three $\mathrm{Zn}^{2+}$-binding sites, as well as the salt bridge that has been proposed to stabilize the dimer (12).

As a first step in producing a structural model consistent with our map, we threaded the sequence for YiiP from $S$. oneidensis onto the X-ray structure of the dimer, which is from E. coli. The C-terminal domain of this starting model was docked, and the transmembrane domains were then manually manipulated to match the corresponding portion of the density map (e.g., Fig. S4). The X-ray structure suggested that the transmembrane domain consisted of two relatively independent elements with limited interactions, namely the M3-M6 pair of helices and the four-helix bundle (M1-M2-M4-M5), which harbors the $\mathrm{Zn}^{2+}$ binding site (12). Indeed, moving these elements within the membrane produced a much better fit for the backbones of the transmembrane helices (Fig. S4).

As a more rigorous approach to fitting the YiiP model to our EM density map and producing an energetically plausible structure, we applied an MD-based flexible fitting (MDFF) method (25). As suggested by our manual fitting and by the interhelical interactions seen in the X-ray structure, the four-helix bundle formed by M1, M2, M4, and M5 was kept rigid and was allowed to move independent from the M3-M6 helix pair as well as from the C-terminal domain. Both IHRSR and FB maps were used for fitting. After $1.5 \mathrm{~ns}$, both simulations reached a plateau in the cross-correlation between the model and the experimental map (0.94 and 0.92 , respectively). The resulting models were very similar to one another, with an rmsd of $2.4 \AA$ for C $\alpha$ atoms (Fig. S4). Given the independence of the two fits, this value is a reasonable statistic for the reliability of the fit, which has been shown to exceed the nominal resolution of the map by as much as 10 -fold (26). In our case, our map's $13-\AA$ resolution is sufficient to accurately position helices, but the orientation of side chains and loops are determined solely by MD simulation. Significantly, the

cross-correlation coefficient was substantially lower (0.54) if the four-helix bundle was not allowed to move relative to M3-M6, supporting the existence of a conformational change relative to the X-ray structure.

Conformational Change Reflects Alternating Access Mechanism of Transport. Comparison of the X-ray structure and the MDFF model representing the cryo-EM map suggests that this conformational change is relevant to the mechanism of YiiP transport. In particular, pivoting of the four-helix bundle relative to M3-M6, which occurs about a point near the cytoplasmic end of M2, appears to switch access of the $\mathrm{Zn}^{2+}$ transport sites from periplasm to cytoplasm (Fig. 3). Specifically, the X-ray structure is characterized by a cavity leading from the transmembrane $\mathrm{Zn}^{2+}$-binding sites to the periplasmic side of the membrane, which has been postulated to be a pathway for binding and releasing the ion $(11$, 12). In our MDFF/EM model, tilting of the four-helix bundle relative to the M3-M6 pair collapsed this periplasm-facing cavity and

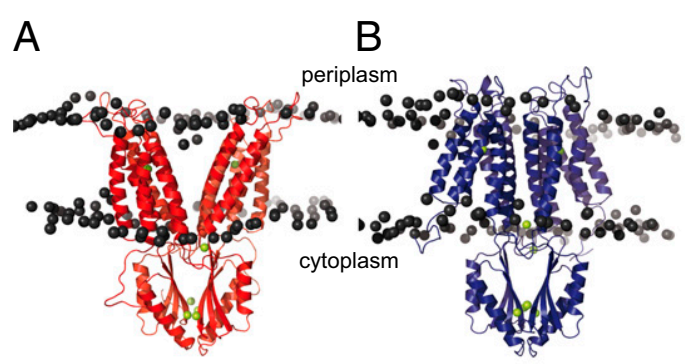

C

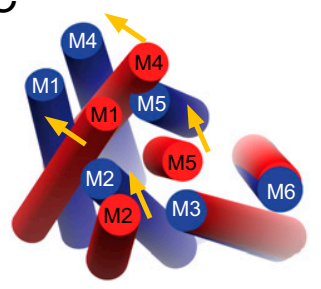

$\mathrm{D}$

E

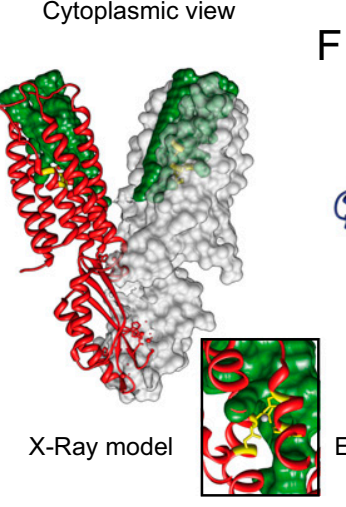

Fig. 3. ( $A$ and $B$ ) Comparison of the $X$-ray and EM models. $X$-ray structures $(A)$ and MDFF/EM structures $(B)$ equilibrated by MD in an explicit DOPG membrane. Comparison of these two structures illustrates the similarity of the C-terminal domains and scissoring movements of the transmembrane helices. ( $C$ and $D$ ) Overlay of the helical bundles from a YiiP monomer showing movement of the M1-M2-M4-M5 bundle relative to the M3-M6 pair, the latter of which has been aligned for this comparison. $(E)$ Spacefilling representation of the X-ray structure of YiiP (PDB ID code $3 \mathrm{H} 90$; in gray), with a cavity (green) leading from the periplasmic side of the membrane. $(F)$ Space-filling representation of the MDFF/EM model (gray) revealing a cavity (green) from the cytoplasmic side of the membrane. The cavities were defined using HOLLOW (37). Residues involved in $\mathrm{Zn}^{2+}$ transport are shown in yellow. (Insets) Transport sites shown at higher magnification and at a slightly different orientation. 
created a new one leading from the cytoplasmic side of the membrane to the $\mathrm{Zn}^{2+}$-binding sites (Fig. 3).

These comparisons suggest that the X-ray and MDFF/EM structures represent the outward-facing and inward-facing conformations that typify the alternating access mechanism $(13,27)$ used by many secondary transporters (Fig. 4). In addition, the transmembrane domains of the monomers diverged by $\sim 30^{\circ}$ in the X-ray structure, but were substantially closer together in the cryo-EM model, producing interactions between the monomers at the periplasmic end of M3, as well as at the cytoplasmic ends of M3 and M6. It is possible that these dimeric interactions within the membrane might coordinate the conformational change of the two monomers.

We used targeted MD simulation to address the dynamics of this conformational change in an explicit DOPG-water environment After adding $\mathrm{Zn}^{2+}$ and equilibrating the inward-facing MDFF/EM structure in the bilayer, we applied a modest steering force to explore the energetic feasibility of making the transition to the outward-facing state. Although the MD structures equilibrated within the membrane environment differed slightly from those determined empirically by EM and X-ray (rmsd of $2 \AA$ and $4 \AA$, respectively; Fig. S6), a switch in the accessibility of the $\mathrm{Zn}^{2+}$ transport sites to water molecules was consistent with the transition from inward-facing to outward-facing states. Thus, this simulation indicates the existence of at least one path for the transition between inward-facing and outward-facing states. Longer equilibration runs are needed to assess the stability of the two conformations in this lipid environment, as well as the influence of ion release and protonation.

We also examined membrane thickness to identify potential mismatches between hydrophobic regions of the protein and the membrane and to assess potential energetic effects on the conformational change. Our analysis considered both total lipids in the simulation, as well as boundary lipids defined to be within $10 \AA$ of the protein, thus revealing a slight thinning of the
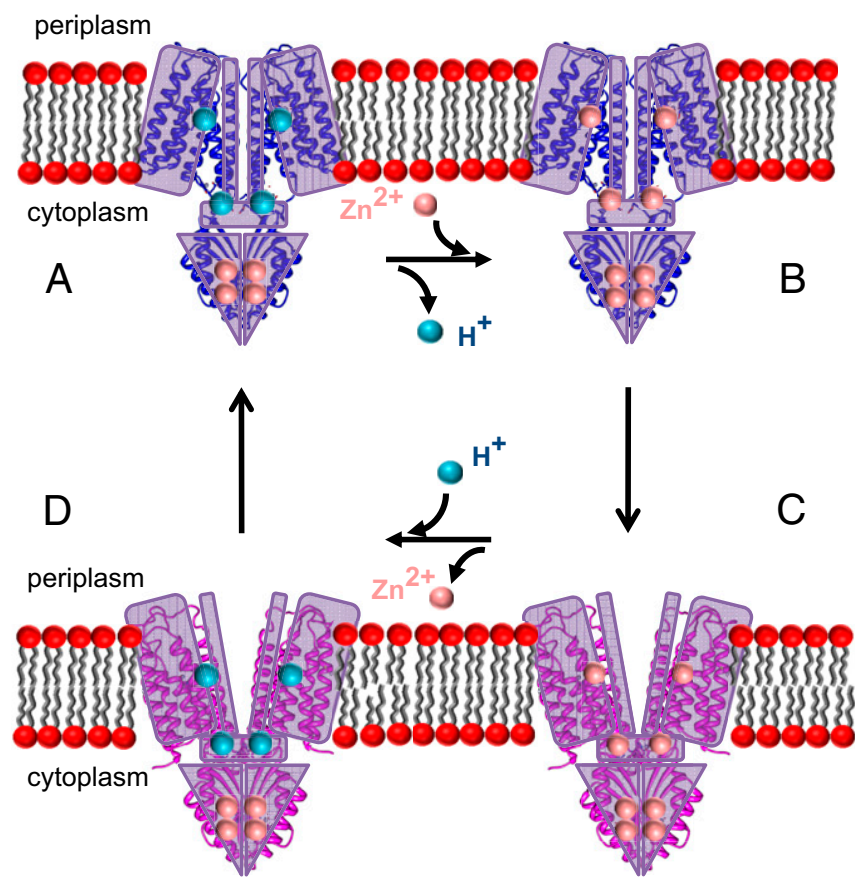

Fig. 4. Alternating access mechanism for $\mathrm{Zn}^{2+} / \mathrm{H}^{+}$antiport by YiiP. This mechanism involves inward-facing $(A$ and $B$ ) and outward-facing $(C$ and $D)$ conformations, both of which are able to bind $\mathrm{Zn}^{2+}$ or $\mathrm{H}^{+}$. The proton motive force provides a driving force for exporting $\mathrm{Zn}^{2+}$ from the cytoplasm. The conformation in $A$ corresponds to that observed in the helical tubes, whereas the conformation in C corresponds to the X-ray structure solved in the presence of $\mathrm{Zn}^{2+}$ membrane next to the protein by $2-3 \AA$ (Fig. S6). Nevertheless, the similarity of membrane thickness in both inward-facing and outward-facing states suggests that there is no significant hydrophobic mismatch that might favor one state over the other.

\section{Discussion}

The structural basis for the alternating access mechanism has been described for several secondary transporter families, which rely on internal symmetries within their protein fold $(27,28)$. In the major facilitator superfamily, typified by GlpT and LacY, a rocker-switch mechanism involves two tandem repeats of a six-helix bundle pivoting about a central ligand-binding site. Transporters with the LeuT fold, such as $\mathrm{Glt}_{\mathrm{Ph}}$ and NhaA, are composed of two inverted repeats that swap conformations in order to switch the accessibility of their substrates. YiiP does not contain internal symmetry, although early work suggested that the transport sites might reside at the interface of the homodimer (20).

The structural comparison presented here indicates that each monomer undergoes a unique conformational change to produce alternating access (Fig. 4). Specifically, the four-helix bundle and the M3-M6 helix pair appear to pivot about the ionbinding site in a manner resembling the rocker-switch mechanism. In our MDFF/EM model, the M3-M6 pairs interact along the dimer interface, suggesting a mechanism for coupling the conformational change of twofold related molecules and offering a possible explanation for why YiiP constitutively dimerizes both in detergent and in the membrane (10).

The energy for inducing the conformational change required for alternating access has been postulated to come from the binding energy of the substrate (29). Thus, it stands to reason that the occupancy of transport sites would influence the conformation adopted by YiiP in the two crystal forms. For the $\mathrm{X}$-ray structure, $\mathrm{Zn}^{2+}$ was included in the mother liquor and was observed to be bound at the transport sites (11), whereas the low $\mathrm{Zn}^{2+}$ concentrations and $\mathrm{pH}$ required to produce the EM crystals suggest that the transport sites were either empty or occupied by protons. Thus, we can conclude that some asymmetry is built into the tertiary structure, such that the protein tends to adopt the periplasmic-facing conformation when saturated with $\mathrm{Zn}^{2+}$. This property would facilitate removal of $\mathrm{Zn}^{2+}$ from the cytoplasm and would enhance coupling with the proton-motive force, consistent with the role of YiiP family members as $\mathrm{Zn}^{2+}$ exporters (2).

The presence of a lipid bilayer in the EM crystals may exert an additional effect on the conformation. The lateral tension, surface charge, and, in the case of our helical crystals, the close packing of YiiP dimers combined with the high curvature (Fig. S7) could serve to pry open the membrane domain to produce the inward-facing conformation. However, the converse is equally plausible, that protonation of the transport site favors the inwardfacing conformation, thus inducing high curvature as the membrane forms during reconstitution. The latter explanation is consistent with our inability to relax the curvature by altering either lipid species or other components of the crystallization buffer.

Of note, the dimeric structure of the C-terminal domain is not affected by this conformational change. This dimer interface is mediated by four $\mathrm{Zn}^{2+}$ ions, and the persistence of this dimer, together with evidence of very-high-affinity $\mathrm{Zn}^{2+}$ binding, suggest that residual $\mathrm{Zn}^{2+}$ still may be bound even under our crystallization conditions. Specifically, isothermal titration calorimetry indicated $\mathrm{Zn}^{2+}$ binding with an affinity too high to be resolved by this technique (19). Furthermore, $\mathrm{Zn}^{2+}$ sites at the interface of C-terminal domains produced significantly higher anomalous X-ray signals than other sites, indicating higher occupancy and thus higher affinity (11).

Finally, when we used EDTA to strip YiiP of its $\mathrm{Zn}^{2+}$, we found that the protein remained stable and tubes grew with moderate EDTA concentrations $(0.5 \mathrm{mM})$, but that prolonged 
incubation at higher concentrations $(5 \mathrm{mM})$ led to protein precipitation. A similar result was previously reported with a $E$. coli homolog (19), suggesting that very-high-affinity metal ion sites may be essential to protein stability. Although the conserved salt bridge at the membrane surface, dubbed the charge interlock, has been implicated in formation of the YiiP dimer (12), it seems possible that the stability of the YiiP dimer also relies on $\mathrm{Zn}^{2+}$ binding to the $\mathrm{C}$-terminal domain. Indeed, structures of $\mathrm{C}$-terminal domain constructs from the homologous CzrB lacking the charge interlock have shown that the precise geometry of the dimer can be achieved simply by binding of $\mathrm{Zn}^{2+}$ at the interface (30). Interestingly, $\mathrm{Zn}^{2+}$ can be removed from these constructs, and although the apo form continues to produce dimers, these dimers adopt variable angles between monomers and demonstrate little interaction across the face of the domain (31). This observation suggests that tethering the monomers with the charge interlock contributes to the high affinity of these $\mathrm{Zn}^{2+}$ binding sites in the dimer, which in turn would maintain a fixed architecture for the C-terminal domains.

An unresolved question is how $\mathrm{Zn}^{2+}$ is delivered to the transport sites. As with $\mathrm{Cu}^{+}$, the levels of free $\mathrm{Zn}^{2+}$ in the cytoplasm have been shown to be virtually zero (32). Unlike $\mathrm{Cu}^{+}$, however, cytoplasmic metallochaperones for delivering $\mathrm{Zn}^{2+}$ to transporters and metalloproteins have not yet been identified (33). Intriguingly, the $\alpha \beta \beta \alpha \beta$ fold of the C-terminal domain from YiiP is related to that of soluble $\mathrm{Cu}^{+}$metallochaperones, and an overlay of the first four elements supports the idea of structural homology. This homology raises the possibility that the C-terminal domain acts as a tethered metallochaperone that binds $\mathrm{Zn}^{2+}$ and transfers it to the transport sites. Intriguingly, ATPdependent $\mathrm{Cu}^{+}$pumps also carry metallochaperone domains on their $\mathrm{N}$ termini, although evidence supports their role in regulation rather than in ion transfer (34).

A regulatory role for the C-terminal domain of YiiP also has been proposed, with $\mathrm{Zn}^{2+}$ causing allosteric changes in that domain that promote binding and transport by the transmembrane domain (12). The hypothesized changes in the C-terminal domain are not supported by our EM structure, however, and the implication that $\mathrm{Zn}^{2+}$ is bound with extremely high affinity makes it difficult to envision how these ions could be transferred to the transport sites. Instead, we favor the idea that $\mathrm{Zn}^{2+}$ acts as a structural element that stabilizes the YiiP dimer, which in turn may facilitate or coordinate the conformational change that accompanies the alternating access mechanism (e.g., by interaction of the M3-M6 pair across the dimer axis). Clearly, more work is needed to clarify both the role of $\mathrm{Zn}^{2+}$ binding by the Cterminal domain and the mechanism by which $\mathrm{Zn}^{2+}$ is delivered to transport sites in a physiological environment that chelates any and all free $\mathrm{Zn}^{2+}$ ions.

\section{Materials and Methods}

Yiip Expression, Purification, and Crystallization. YiiP (Q8E919) from S. oneidensis was expressed in a 3-L culture of $E$. coli, solubilized with $30 \mathrm{mM}$ $\mathrm{n}$-dodecyl $\beta$-D-maltoside, and purified by nickel-nitrilotriacetic acid affinity chromatography. After cleaving the deca-histidine tag with tobacco etch virus protease, the protein was further purified by size-exclusion chromatography, producing a pooled fraction with $\sim 6 \mathrm{mg}$ in $3 \mathrm{~mL}$, which was used for 2D crystallization without further concentration. For crystallization, YiiP was mixed with detergent-solubilized DOPG at a lipid:protein mass ratio of $1: 1$ and dialyzed against $20 \mathrm{mM} \mathrm{N}$-[tris(hydroxymethyl)methyl]-2-

1. Degtyarenko K (2000) Bioinorganic motifs: Towards functional classification of metalloproteins. Bioinformatics 16(10):851-864.

2. Blencowe DK, Morby AP (2003) Zn(II) metabolism in prokaryotes. FEMS Microbiol Rev 27(2-3):291-311.

3. Zhao H, Eide D (1996) The yeast ZRT1 gene encodes the zinc transporter protein of a high-affinity uptake system induced by zinc limitation. Proc Natl Acad Sci USA 93(6): 2454-2458.

4. Nies DH, Silver S (1995) Ion efflux systems involved in bacterial metal resistances. J Ind Microbiol 14(2):186-199. aminoethanesulfonic acid (pH 7.0), $5 \mathrm{mM} \mathrm{MgCl}, 100 \mathrm{mM} \mathrm{NaCl}$, and $5 \mathrm{mM}$ $\mathrm{NaN}_{3}$. Crystals started growing after 1-2 d and were generally harvested after 5-7 d. To test the effect of $\mathrm{Zn}^{2+}$ on crystallization, citrate and $\mathrm{N}$-(2acetamido)iminodiacetic acid were used to buffer free $\mathrm{Zn}^{2+}$ concentrations between $10 \mathrm{nM}$ and $1 \mathrm{mM}$. All experimental procedures are described in more detail in SI Materials and Methods.

Reconstitution and Transport Assays. Reconstitution and stopped-flow transport assays were conducted using procedures described by Wei and Fu (8). Purified YiiP was reconstituted in E. coli polar lipids at a protein:lipid mass ratio of $1: 2,000$ by dialysis against $20 \mathrm{mM}$ Hepes $\left(\mathrm{pH} \mathrm{6.5)}\right.$ and $50 \mathrm{mM} \mathrm{K}_{2} \mathrm{SO}_{4}$ at $4{ }^{\circ} \mathrm{C}$ for $4 \mathrm{~d}$. Proteoliposomes were loaded with the $\mathrm{Zn}$-sensitive fluorophore FluoZin- 1 by freeze-thaw, and then extruded through $0.4-\mu \mathrm{m}$ polycarbonate membranes. After removal of free FluoZin-1 with a desalting column, liposomes were mixed with solutions containing either $\mathrm{ZnCl}_{2}(20 \mu \mathrm{M}-8 \mathrm{mM})$ or $\mathrm{CdCl}_{2}(20 \mu \mathrm{M}-4 \mathrm{mM})$ using a stopped-flow apparatus coupled to a fluorimeter with excitation at $490 \mathrm{~nm}$ and emission at $525 \mathrm{~nm}$. Protein-free liposomes were used to measure background, and fluorescence signals were normalized to those produced with $4 \% \beta$-octyl-D-glucoside and either $8 \mathrm{mM} \mathrm{ZnCl}_{2}$ or $4 \mathrm{mM}$ $\mathrm{CdCl}_{2}$. Initial rates were fitted with the Hill equation, and $\mathrm{pH}$ dependence was measured by incubating liposomes in buffers at $\mathrm{pH} 6.5-9$ overnight before the transport assays were conducted.

EM, Image Analysis, and Computational Methods. Images of frozen-hydrated crystals were recorded on film and digitized with an effective pixel size of $2.73 \AA$ on the specimen scale. Helical symmetry was deduced by assigning Bessel orders to layer lines in the Fourier transform and by calculating corresponding values for the azimuthal rotation $(\Delta \phi)$ and rise $(\Delta z)$ relating one subunit to the next. IHRSR reconstruction was performed in SPARX (35), taking into account the D3 symmetry of these helical crystals. The IHRSR cycle was iterated until the Euler angle assignments for individual segments ceased to change. FB reconstruction was performed using EMIP (http://cryoem.nysbc.org/EmIP.html), which makes use of the strategies developed by Beroukhim and Unwin $(23,36)$.

To determine the correct hand for the map, asymmetric units (shown in gray in Fig. 3) were extracted from maps generated with alternate handedness and compared with the X-ray structure of YiiP (Fig. S8). For MDFF (25), a homology model of YiiP from S. oneidensis was built with MODELER 9v7 using the X-ray crystal structure of YiiP from E. coli (PDB ID code 3H90) as a template. An external potential was defined as $\zeta\left(1-\varphi / \varphi_{\text {Max }}\right)$, where $\varphi / \varphi_{\operatorname{Max}}$ is the normalized density value from the map and $\zeta$ is a scaling factor. An allatom simulation was conducted in vacuo using the CHARMM force field. In the first step, with $\zeta=1$, harmonic potentials of elastic constant $\mathrm{k}=500 \mathrm{kcal} /$ (mol. $\AA^{2}$ ) were applied to (i) the four-helix bundle formed by M1, M2, M4, and M5; (ii) the M3 and M6 helices at the dimeric interface; and (iii) the C-terminal cytoplasmic domain for $0.5 \mathrm{~ns}$. Then the harmonic restraints were released, and an additional 1-ns fitting was performed using a scaling constant of $\zeta=$ 0.3. Targeted MD was performed in an explicit DOPG lipid bilayer solvated by TIP3P water. After a 3-ns equilibration, the $C_{\alpha}$ rmsd for the transmembrane domain of the MD model relative to the $\mathrm{X}$-ray structure was biased toward 0 for $1.0 \mathrm{~ns}$ using a harmonic force with an elastic constant of 1,500 kcal/ $\left(\mathrm{mol} \cdot \AA^{2}\right)$. After steering, the system was re-equilibrated without biases or restraints for an additional 3 ns.

ACKNOWLEDGMENTS. We thank James Love and the Membrane Protein Expression Laboratory of the New York Consortium on Membrane Protein Structure for identification and initial screening of the YiiP homolog as a suitable target for structural analysis. We also thank Dax Fu for help with the transport assay. Computer simulations were run on resources available through the Scientific Computing Facility of the Icahn School of Medicine at Mt. Sinai, and were supported in part by the National Science Foundation through TeraGrid advanced computing resources provided by Texas Advanced Computing Center Grant TG-MCB080109N. Funding for this work was provided by National Institutes of Health Grants U54 GM94598 and R01 GM095747.

5. Palmiter RD, Findley SD (1995) Cloning and functional characterization of a mammalian zinc transporter that confers resistance to zinc. EMBO J 14(4): 639-649.

6. Gaither LA, Eide DJ (2001) Eukaryotic zinc transporters and their regulation. Biometals 14:251-270.

7. Grass G, et al. (2005) FieF (YiiP) from Escherichia coli mediates decreased cellular accumulation of iron and relieves iron stress. Arch Microbiol 183(1):9-18.

8. Wei Y, Fu D (2005) Selective metal binding to a membrane-embedded aspartate in the Escherichia coli metal transporter YiiP (FieF). J Biol Chem 280(40):33716-33724. 
9. Hoch E, et al. (2012) Histidine pairing at the metal transport site of mammalian ZnT transporters controls $\mathrm{Zn2}^{+}$over $\mathrm{Cd}^{+}$selectivity. Proc Natl Acad Sci USA 109(19): 7202-7207.

10. Wei Y, Li H, Fu D (2004) Oligomeric state of the Escherichia coli metal transporter YiiP. J Biol Chem 279(38):39251-39259.

11. Lu M, Fu D (2007) Structure of the zinc transporter YiiP. Science 317(5845):1746-1748.

12. Lu M, Chai J, Fu D (2009) Structural basis for autoregulation of the zinc transporter YiiP. Nat Struct Mol Biol 16(10):1063-1067.

13. Jardetzky O (1966) Simple allosteric model for membrane pumps. Nature 211(5052) 969-970.

14. Ubarretxena-Belandia I, Stokes DL (2012) Membrane protein structure determination by electron crystallography. Curr Opin Struct Biol 22(4):520-528.

15. Love J, et al. (2010) The New York Consortium on Membrane Protein Structure (NYCOMPS): A high-throughput platform for structural genomics of integral membrane proteins. J Struct Funct Genomics 11(3):191-199.

16. Kim C, et al. (2010) An automated pipeline to screen membrane protein 2D crystallization. J Struct Funct Genomics 11(2):155-166.

17. Vink M, Derr K, Love J, Stokes DL, Ubarretxena-Belandia I (2007) A high-throughput strategy to screen 2D crystallization trials of membrane proteins. J Struct Biol 160(3) 295-304.

18. Hu M, et al. (2010) Automated electron microscopy for evaluating two-dimensional crystallization of membrane proteins. J Struct Biol 171(1):102-110.

19. Chao Y, Fu D (2004) Kinetic study of the antiport mechanism of an Escherichia coli zinc transporter, ZitB. J Biol Chem 279(13):12043-12050.

20. Wei Y, Fu D (2006) Binding and transport of metal ions at the dimer interface of the Escherichia coli metal transporter YiiP. J Biol Chem 281(33):23492-23502.

21. Chao Y, Fu D (2004) Thermodynamic studies of the mechanism of metal binding to the Escherichia coli zinc transporter YiiP. J Biol Chem 279(17):17173-17180.

22. Egelman EH (2000) A robust algorithm for the reconstruction of helical filaments using single-particle methods. Ultramicroscopy 85(4):225-234.

23. Diaz R, Rice WJ, Stokes DL (2010) Fourier-Bessel reconstruction of helical assemblies. Methods Enzymol 482:131-165.
24. Lewis BA, Engelman DM (1983) Lipid bilayer thickness varies linearly with acyl chain length in fluid phosphatidylcholine vesicles. $J$ Mol Biol 166(2):211-217.

25. Trabuco LG, Villa E, Mitra K, Frank J, Schulten K (2008) Flexible fitting of atomic structures into electron microscopy maps using molecular dynamics. Structure 16(5): 673-683.

26. Rossmann MG (2000) Fitting atomic models into electron-microscopy maps. Acta Crystallogr D Biol Crystallogr 56(Pt 10):1341-1349.

27. Forrest LR, Krämer R, Ziegler C (2011) The structural basis of secondary active transport mechanisms. Biochim Biophys Acta 1807(2):167-188.

28. Boudker O, Verdon G (2010) Structural perspectives on secondary active transporters. Trends Pharmacol Sci 31(9):418-426.

29. Klingenberg M (2005) Ligand-protein interaction in biomembrane carriers: The induced transition fit of transport catalysis. Biochemistry 44(24):8563-8570.

30. Cherezov V, et al. (2008) Insights into the mode of action of a putative zinc transporter CzrB in Thermus thermophilus. Structure 16(9):1378-1388.

31. Higuchi T, Hattori M, Tanaka Y, Ishitani R, Nureki O (2009) Crystal structure of the cytosolic domain of the cation diffusion facilitator family protein. Proteins 76(3): 768-771.

32. Outten CE, O'Halloran TV (2001) Femtomolar sensitivity of metalloregulatory proteins controlling zinc homeostasis. Science 292(5526):2488-2492.

33. Tottey $S$, et al. (2008) Protein-folding location can regulate manganese-binding versus copper- or zinc-binding. Nature 455(7216):1138-1142.

34. Argüello JM, González-Guerrero M, Raimunda D (2011) Bacterial transition metal P(1B)-ATPases: Transport mechanism and roles in virulence. Biochemistry 50(46): 9940-9949.

35. Behrmann E, et al. (2012) Real-space processing of helical filaments in SPARX. J Struct Biol 177(2):302-313.

36. Beroukhim R, Unwin N (1997) Distortion correction of tubular crystals: Improvements in the acetylcholine receptor structure. Ultramicroscopy 70(1-2):57-81.

37. Ho BK, Gruswitz F (2008) HOLLOW: Generating accurate representations of channel and interior surfaces in molecular structures. BMC Struct Biol 8:49. 